\title{
The Expression Mode of Chinese Characters' Dynamic Font Design*
}

\author{
Lu Zheng \\ Nanjing University of Aeronautics and Astronautics \\ Nanjing, China
}

\author{
Wenxi Zhong \\ Nanjing Audit University Jinshen College \\ Nanjing, China
}

\begin{abstract}
With the rapid development of digital media technology, the field of visual communication design tends to be diversified. The dynamic design of Chinese character fonts continues to evolve with the rapid development of new technologies. Chinese characters, as the theme of Chinese culture, also have more intense emotions and distinctive personalities. Visual presentation. The use of a variety of creative forms is more conducive to the development of the dynamic font design of Chinese characters, and the method of expression is more exciting along with the changes in the design art form.
\end{abstract}

Keywords-Chinese characters; dynamic font design; digitization

\section{INTRODUCTION}

Chinese characters being horizontal and vertical are bone, flying left-falling and right-falling strokes are blood, which are highly summarizing the spirit of Chinese characters, it is the information carrier of conveying and spreading Chinese culture, the font of Chinese characters is also an inevitable and important part of design, which has largely wide development space. With the rapid development of new media spreading route, the continuous update and expansion of design theory, the spreading and promotion of Chinese culture cannot be separated from Chinese characters, and the font design as the most link in the visual design, the dynamic font design of Chinese characters continue to be updated with the rapid development of new technology. The expression of dynamic font design of Chinese characters is the process of the mutual transformation of characters and graphics through the change of time and space. For static font design, dynamic font design enables font design contents to be more pictorial through the changes of spatial and temporal dimensions, which makes the design performance more vivid and diversified.

The dynamic changes are realized via time changes, Time is a ruler that can measure the change because it describes change. If there is no change, there will be no time. ${ }^{1}$ Time can change the characteristics of fonts in space, and also provide dynamic font design with more possibilities. And the dynamic Chinese character font is visual expression, which is more vital, also has a stronger emotion and a

*Fund project: Postgraduate Research \& Practice Innovation Program of Jiangsu Province in 2017 of "the Expression Mode of Chinese Characters' Dynamic Font Design”, project number: kfjj20171108 distinctive personality.

The combination of Chinese characters' graphic vision design and digital media technology produces Chinese characters' dynamic font, this new form of design language. Its timeliness, space, narrativity and emotion make Chinese characters' font more vital, and it can better reflect the personalized and emotional font in the design of visual expression effect.

\section{EMOTION EXPRESSION IN THE DYNAMIC FONT DESIGN OF CHINESE CHARACTERS}

The most important difference between dynamic and static font, from two perspectives, is the change of time and space dimensions, the dynamic font describes the change of space. On the other hand, picture changes also reflect time changes. Dynamic font design uses the change of font in the main space and presents the movement and change process of font through the deconstruction and arrangement of font In fact, for the consciousness of the people, in order to achieve an effective cognition, font on the dynamic scheduling needs to be performed in the space at a speed as slow as possible, by doing so, the font information can be conveyed more accurately, also can strengthen people's memory for this dynamic font, it can be said that time changes could reflect the font's emotion changes.

Shi Wenya's Research on Characters Design in Information Design of New Media talked about the relationship between character information and the audience, the design of dynamic Latin characters can effectively express the talker's tone, character's personality and emotion, give the film text a rich emotional character. Compared with static text, it can provide the audience with different types of presentation and more clearly guide or manipulate the audience's attention.

In fact, in the famous director Hitchcock's film North by Northwest, as shown in "Fig. 1" and later Asylum Seekers, as shown in "Fig. 2", dynamic text technology was adopted early in the presentation of the film's opening cast list. This technology broke the silence of the text in the original space through the aid of graphics, and then deconstructed the text at a slow speed. It was not difficult to see that the director expected to create an emotional tone related to the film's plot with the opening list of actors, instead of simply conveying the message of the actors 


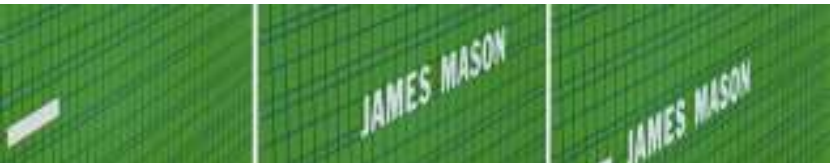

Fig. 1. The opening of film North by Northwest.


Fig. 2. The opening of film Asylum Seekers.

In the opening of the domestic director Jiang Wen's Let the Bullets Fly, a bullet directly crosses the four characters Let the Bullets Fly, vividly interprets the connotation of these four Chinese characters. The bullets boring leads to the space changes to the original bullet character, the dynamic change of bullet graphics makes the font change, as shown in "Fig 3". The rapid speed of the bullet echoes the original meaning of the four Chinese characters, and it also highlights the atmosphere of the ups and downs of the plot, which complements each other. The intense transformation brings out the connotation of the words and the strong resonance of the audience's emotions, which then lays the foundation of the tone of the film itself.
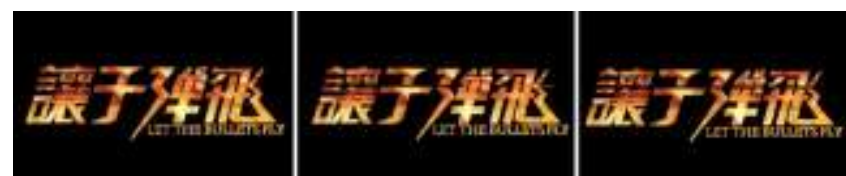

Fig. 3. The opening of film Let the Bullets Fly.

Chinese characters font itself has the characteristics of imagery on the vision, the imagery makes Chinese characters beyond alphabetic writing thinking's linear structure, building a three-dimensional thinking space, due to the increase of the dimension, the dynamic relationship of structure is more complex, the dynamic changes of the shape is more rich, this will probably strengthen the rhythm of dynamic font design of Chinese characters, thus the emotion can be expressed more clearly and exquisitely.

\section{VITALITY EXPRESSION IN THE DYNAMIC FONT DESIGN OF CHINESE CHARACTERS}

In 2016, Taiwanese designer He Ting 'an released the dynamic font design work named "Motion Type Project", in which he adopted the font Type provided by Monotype, as shown in "Fig 4", used a deconstructive way to analyze Chinese characters, deducted the original features of each Chinese character through dynamic changes, and endowed each Chinese character with different vitality.

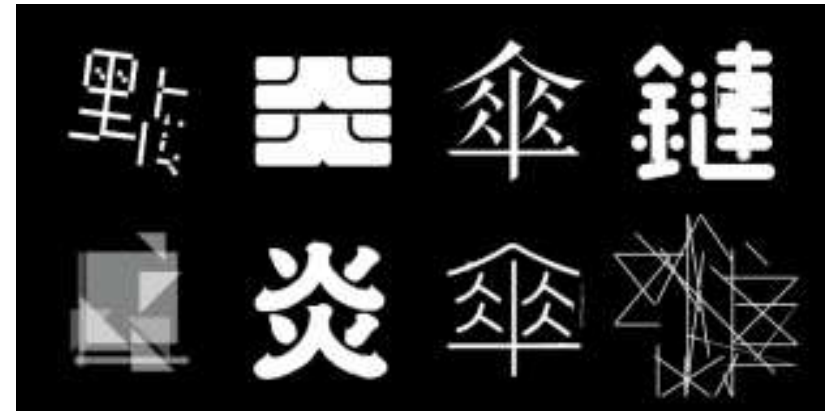

Fig. 4. He Ting'an's font design.

Such as “伞(means umbrella in English)” this Chinese character, the designer made the dynamic recombination and added the special effect of rain flow through deconstructing strokes, vividly interpreted the original meaning, use and scene of the umbrella, as if presenting a vivid rain scene for the audience, completed the information transmission of Chinese characters, added the interestingness of the words, and expressed the characters' meaning vividly and accurately, as shown in "Fig 5". The smooth sliding between fonts, rapid jitter and lively bounce are totally different to the audience's inner feelings. Therefore, different modes of movement can endow dynamic fonts with completely different vitality, which is different from static vision. The Chinese characters with vitality make the whole meaning of the word easier to be interpreted and can better convey the information to the audience while understanding the meaning.
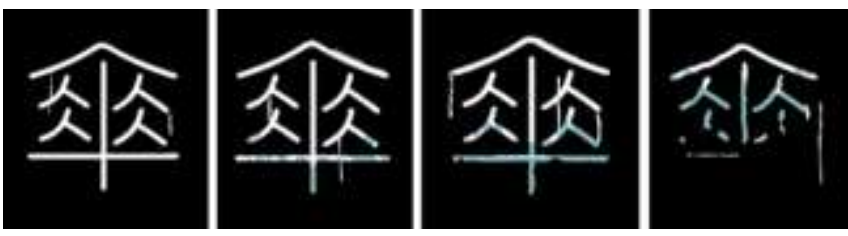

Fig. 5. He Ting' an's font design “伞(means umbrella in English)”.

\section{PERSONAL EXPRESSION IN THE DYNAMIC FONT DESIGN OF CHINESE CHARACTERS}

The dynamic font created by the designers from 17 countries for the Shanghai design exhibition in 2014, as shown in "Fig 6" caused quite a shock in the field of dynamic font design of Chinese characters. Through their own understanding of the characteristics of Shanghai urban culture, the designers carried out dynamic font design with " 上海(Shanghai)" as the theme, as shown in "Fig 6". The designer $\mathrm{Xu}$ Yibing who was Shanghai native, created a dynamic font design work by using the "dot" element with a sense of fluctuation. In the work, dots of different sizes and colors were formed into abstract "上海 (Shanghai)", these two Chinese characters, through time changes in the picture, these dots were regularly pulsating in the picture, which caused a dynamic effect. The dots of different sizes made the picture have a sense of hierarchy and space, and the spatial relationship between the dots made the picture have a strong sense of future and full of vitality and vitality. Through the change of time, a dynamic graph was formed in the space, which endowed the wave texture of "上海(Shanghai)", and 
added fashionable dot elements, the dynamic font design of " 上海(Shanghai)" demonstrates the unique vitality of Shanghai and also presents the personality charm of font dynamic design. It makes the dynamic Chinese character font design different from static, also have the personality of the character.

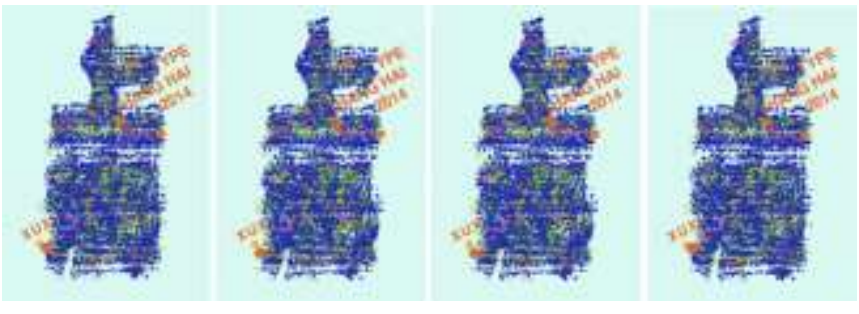

Fig. 6. Xu Yibing’s font design “上海(Shanghai)”.

Baidu search page also used dynamic font to present the personalized search logo, as shown in "Fig. 7", March 18 is the Earth Hour Day, the designers design by taking global warming, low-carbon life, avoiding the waste, refusing wildlife products and green consumption as the entry point, combining with pinyin bai and baidu in Chinese typeface, among which the character of Bai skillfully integrates the polar bear's helpless standing on the ice floes, buses and other elements into the font design, and also reminds and guides the audience to pay attention to global warming. With the $60+$ sign proposed by the world wild life fund and the mutual changes of the panda and octopus that respectively represents the earth's land and marine life echoing, the lower part “叉(means and in English)" of the character 度 is designed to a style of residential buildings, being bright and dim reflects the concept of energy saving and environmental protection, also allows the audience clearly understand the Earth Hour Day, this global day theme.

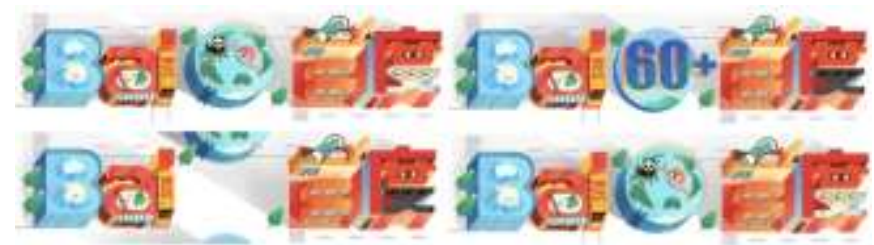

Fig. 7. Baidu search interface animation.

The above two cases fully show that the personal dynamic design of Chinese characters is often customized, having distinct characteristics and personalized style, the expressive force of font can make the audience feel refreshing, dynamic design of Chinese characters under the thematic, proposition context can highlight the personalized design feature of the font.

\section{DIVERSITY OF DYNAMIC FONT DESIGN OF CHINESE CHARACTERS}

\section{A. Application of Digital Technology in Dynamic Font Design of Chinese Characters}

Whether the dynamic font of Chinese characters is designed through interactive design or video image and other technical forms of Chinese characters' dynamic font design, digital media technology is the basic guarantee to achieve the effect of works. The application of digital technology is undoubtedly the basic premise of future design and also an important technical means of dynamic font design of Chinese characters. Without technical support, the effect and form of dynamic font design is difficult or impossible to be achieved. If the two-dimensional font design is regarded as a relatively simple communication medium, then the dynamic font design has the characteristics of media diversification. For example: computer software technology, visual network activities, digital media, video image, information acquisition technology and interactive experience, and other Chinese characters' dynamic font design.

\section{B. Diversified Design Concepts and Styles}

The equations are an exception to the prescribed specifications of this template. You will need to determine whether or not your equation should be typed using either the Times New Roman or the Symbol font (please no other font). To create multileveled equations, it may be necessary to treat the equation as a graphic and insert it into the text after your paper is styled. The application of new digital technology in the dynamic design of Chinese characters is bound to give birth to new aesthetic concepts and audience demands. Under the influence of aesthetics and demands, people are eager to see more novel design forms rapidly. Meanwhile, the novel design concepts and styles also change the production and life of audience.

The designer Jiang Nanli, chooses the song title of his favorite singer Tian Fuzhen for the font design, and makes a new attempt by combining the late software of adobe, AE with Motion Graphic (dynamic Graphic design). "Saying" this work, extracts the lyrics, arranges and combines on a yellow background, the simulated wind is blowing, blows out the font and the tree on the left fork, the fork tree is arranged and combined to form the font 念念 (means saying in English) and 有词 (means words in English) are skillfully arranged, the whole picture is fluent, comfortable graphic symbol and font supplement each other, and skillfully matching with the lyrics' artistic conception, as shown in "Fig. 8". The designer also puts the popular QR code into the picture, and the song can be heard by scanning the code, creating an interaction with the audience that breaks the twodimensional space.

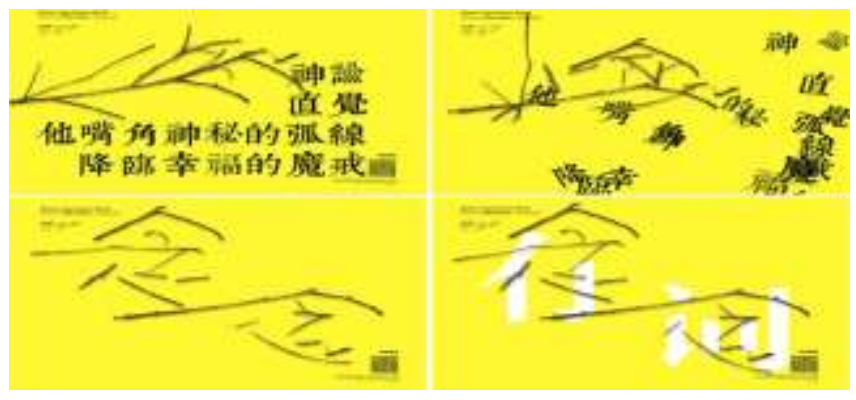

Fig. 8. Jiang Nanli’s font design 念念 (means saying in English) and 有词 (means words in English). 
Digital technology and design form continue to exist through the continuous integration and convergence of design results. Character itself has the features of conveying communication message and displaying design integrity concept. Driven by new digital media, the font design featuring character form should have better perception ability, and quickly give a reasonable response strategy to digital demand, thus bringing the design audience a happy and fresh feeling. This quick response strategy is not only about character communication, but also about an innovative update of the font. This update is mainly about character structure, character arrangement and the possibility of using new materials.

No matter in the design concept or form, the expression form of the future Chinese characters' dynamic font and the transmission medium have diversified features, and the expression forms such as interactive experience and video image are not fixed and single. The boundary between the form of contemporary art and modern design tends to be blurred. The blur of the design boundary is determined by the era development. The dynamic font design of Chinese characters can use these creative forms for reference and inspire each other in the expression form, so as to facilitate the expansion of the thoughts of the dynamic font of Chinese characters and make the methods of expression follow the diversified forms of design art to become rich and wonderful.

\section{CONCLUSION}

The dynamic font design of Chinese characters is oriented towards the future, which is still a new field today. The era development definitely determines its future development potential.

Dynamic font design is constantly enriched by the development of film and animation, and it is the continuation of font communication in the dynamic media environment. Font design has added new meaning in time and space dimension, which also makes the characters bear more information and produce stronger visual impact. As a comprehensive art in the field of digital technology, dynamic font design integrates animation design, sound effect design, interactive design, visual effect design, characters and graphics design and other art forms, dynamic interactive character design can express richer meaning and emotion through movement and sound.

Dynamic font design of Chinese characters is inseparable from China's own cultural connotation, and its own cultural background and the fusion of art form, makes the formation of dynamic font design of Chinese characters different from the independent visual form of other dynamic character font design, draws the philosophical connotation of traditional Chinese art and spirit nutrition, refers to the performance methods of the traditional art, and observes the design of Chinese characters and the relationship between the contemporary art and the future new media art from all perspectives at the same time, this is the future development direction of the dynamic font design of Chinese characters.

\section{REFERENCES}

[1] By Rudolf Arnheim. Translated by Teng Shouyao. Art and visual perception. M.Sichuan: Sichuan People's Publishing House, 2006.

[2] Li Guanlin. Preliminary study on space design of Chinese characters.J.Tangdu Journal 2010, (26).

[3] Tao Lin. Research on the dynamic design of the font .D. Nanjing Donghua University, 2007.

[4] Gao Xiaoming. The new trend of Chinese characters' art vision in digital age .J.Luo he Vocational Technology College Journal. 2010, (9).

[5] By Rudolf Arnheim. Translated by Teng Shouyao. Visual thinking. M.Sichuan: Sichuan People's Publishing House, 2006. 